\title{
tic\&société
}

Vol. 2, $n^{\circ} 2$ | 2008

Société de l'information?

\section{Du logiciel libre aux théories de l'intelligence collective}

Sébastien Broca

\section{(2) OpenEdition \\ Journals}

Édition électronique

URL : http://journals.openedition.org/ticetsociete/451

DOI : 10.4000/ticetsociete.451

Éditeur

Association ARTIC

Référence électronique

Sébastien Broca, « Du logiciel libre aux théories de l'intelligence collective », tic\&société [En ligne], Vol. 2, n 2 | 2008, mis en ligne le 28 mars 2009, consulté le 19 avril 2019. URL : http:// journals.openedition.org/ticetsociete/451; DOI : 10.4000/ticetsociete.451 


\section{Du logiciel libre aux théories de l'intelligence collective}

\section{Sébastien BROCA}

Allocataire de recherche au CETCOPRA, Université Paris 1

17 rue de la Sorbonne

75005 Paris

brocasebastien@yahoo.fr

Sébastien Broca est allocataire de recherche au Cetcopra (Université Paris 1), où il réalise son doctorat de sociologie sous la direction de Philippe Breton. Après avoir travaillé sur l'opposition existant entre l'imaginaire d'Internet et le projet démocratique, il consacre actuellement ses recherches à « l'intelligence collective » en tant qu'utopie sociale postmoderne. II a participé à l'ouvrage collectif /La connaissance dans les sociétés techniciennes/ dirigé par S. Craipeau, G. Dubey, B. Paulré et P. Musso (à paraître en 2009). 


\title{
Du logiciel libre aux théories de l'intelligence collective
}

\begin{abstract}
Résumé
Cet article interroge la manière dont le mouvement du logiciel libre se trouve constitué dans de nombreux discours en modèle d'avant-garde de transformations sociales globales. Je montre ainsi comment l'on passe d'une pratique singulière, mise en œuvre par les communautés du libre, à des théories sociologiques, économiques ou philosophiques, qui s'en inspirent largement. Je m'appuie pour ce faire sur les ouvrages récents de Pekka Himanen, Yann Moulier Boutang, Antonio Negri et Michael Hardt. J'essaie ensuite de mettre en lumière certaines difficultés relatives aux démarches de ces auteurs : généralisation abusive à partir de l'exemple du logiciel libre, et oubli des spécificités des divers domaines de la vie sociale.
\end{abstract}

Mots clés : logiciel libre, intelligence collective, collaboration, Internet, démocratie.

\begin{abstract}
In this paper, I discuss the way the free software movement is often described as the model of upcoming global social changes. Considering recent writings of Pekka Himanen, Yann Moulier Boutang, Antonio Negri and Michael Hardt, I show how the open model experienced by free software communities has inspired several theories in the fields of sociology, economy and philosophy. I then try to highlight some controversial aspects of these theories : faulty generalization of the example of free software, and failure to take into account the specificity of each part of our social life.
\end{abstract}

Keywords : free software, collective intelligence, collaboration, Internet, democracy.

\section{Resumen}

Este artículo examina la manera en que, a menudo, se ha descrito el movimiento del software libre como el modelo vanguardista de las transformaciones sociales globales. Muestra como se pasa de una práctica singular -como es la llevada a cabo por las comunidades de software libre-, a teorías sociológicas, económicas y filosóficas, que se inspiran de dicho modelo. Para ello se recurre a las obras recientes de Pekka Himanen, Yann 


\section{Sébastien BROCA}

Moulier Boutang, Antonio Negri y Michael Hardt. Finalmente, se explicitan algunas dificultades caracterizan a estas teorías, como son la generalización abusiva del ejemplo especifico del software libre, y el olvido de las especificidades presentes en los diferentes campos de nuestra vida social.

Palabras clave : software libre, inteligencia colectiva, colaboración, Internet, democracia. 
Du logiciel libre aux théories de l'intelligence collective

\section{Introduction}

Depuis une quinzaine d'années, le mouvement du logiciel libre a connu un développement fulgurant, mettant fortement en question la prééminence des logiciels propriétaires développés par les grandes entreprises du secteur informatique. Dans le même temps, certains intellectuels ont érigé ces bouleversements en symboles de transformations sociales plus générales, attendues ou espérées. En témoignent les occurrences nombreuses des références à la « démocratie open source », à « l'économie open source », voire à la « société open source». Cette tendance à faire du mouvement du logiciel libre un des laboratoires où se préparerait la société du futur semble devoir nous interpeller à plus d'un titre. Elle incite d'une part à s'interroger sur le bien-fondé d'une démarche intellectuelle prenant appui sur une pratique spécifique, pour fonder un discours théorique à valeur générale et/ou prospective. Elle invite d'autre part à mener une réflexion critique sur les nouvelles grilles d'analyse, censées rendre compte des spécificités de notre époque.

Nous commencerons ainsi par retracer brièvement l'histoire du mouvement du logiciel libre, et par en rappeler les enjeux. Nous essaierons ensuite de montrer comment, sous l'effet d'un double mouvement d'idéalisation des pratiques et de généralisation de leur portée, les communautés du libre se trouvent présentées, dans un certain nombre de discours contemporains, comme porteuses d'un véritable modèle social d'avant-garde. Nous nous efforcerons aussi d'interroger les limites de ces discours, qui tendent à observer notre époque à travers le prisme unique du développement de pratiques de collaboration horizontales médiatisées par Internet.

\section{Histoire et enjeux du mouvement du logiciel libre}

\subsection{Une brève histoire du libre}

Le mouvement du logiciel libre se comprend comme une réaction aux changements intervenus dans l'industrie informatique au tournant des années 1970-1980, au moment où apparaît l'ordinateur personnel. Jusqu'à cette date, les utilisateurs et les programmeurs sont souvent les mêmes personnes, et à quelques exceptions près, ils peuvent librement modifier les logiciels, quand bien même ceux-ci sont soumis au copyright. Comme l'explique Eben Moglen, 


\section{Sébastien BROCA}

dans la pratique, les logiciels pour supercalculateurs étaient développés de manière coopérative par le constructeur de matériel dominant et par ses utilisateurs techniquement compétents [...] (Moglen, 2001, p. 160).

La situation évolue au début des années 1980. De nombreuses sociétés informatiques décident alors d'imposer des logiciels propriétaires, de privatiser du code auparavant libre, et de soumettre leurs informaticiens à des clauses de confidentialité ${ }^{1}$.

Pour mesurer ces bouleversements, il faut savoir qu'un logiciel se présente sous deux formes. La première, dite version exécutable ou compilée, est écrite en «binaire ». C'est celle qui est lue par l'ordinateur, et elle n'est pas compréhensible pour un humain. La deuxième en revanche - appelée code source - n'est pas fonctionnelle, mais peut être appréhendée comme du «commentaire ». Écrite dans un langage de programmation compréhensible, elle explique aux développeurs comment fonctionne le programme. L'accès au code source est donc indispensable à qui veut opérer des modifications sur un logiciel. Or, c'est précisément cet accès qui se trouve fortement restreint par le mouvement de privatisation survenu au début des années 1980, qui empêche donc la communauté des informaticiens de collaborer pour améliorer les programmes.

C'est dans ce contexte que Richard Stallman, alors informaticien au laboratoire d'intelligence artificielle du MIT (Massachusetts Institute of Technology), décide en 1983 d'entreprendre la programmation d'un système d'exploitation entièrement «libre ». II nomme celui-ci GNU (GNU is Not Unix), marquant ainsi sa volonté de se démarquer des nouvelles pratiques de l'informatique commerciale et de retrouver l'esprit coopératif d'antan. En 1985, il crée la Free Software Foundation pour faciliter le financement et le développement du projet. Les principes du logiciel libre sont formalisés en janvier 1989 dans la licence publique générale (GPL - General Public License). Celle-ci garantit aux utilisateurs quatre «libertés »: liberté d'utiliser le logiciel, liberté de le copier, liberté de le modifier (ce qui implique l'accès au code source), et liberté de le distribuer (y compris dans des versions modifiées). Tout

\footnotetext{
1 L'arrivée de nouveaux acteurs industriels est symbolique de ces bouleversements. Un exemple emblématique est la création de la société Sun en 1982 par d'anciens étudiants de Stanford et de Berkeley. Celle-ci privatise en effet de nombreux logiciels du monde Unix.
} 
Du logiciel libre aux théories de l'intelligence collective

logiciel respectant l'ensemble de ces «libertés " peut dès lors être considéré comme un « logiciel libre ${ }^{2}$.

Au début des années 1990, le projet GNU a abouti à l'écriture d'un système d'exploitation presque complet. Presque, car le noyau du système ${ }^{3}$ fait encore défaut. Intervient alors la deuxième grande figure de l'histoire du logiciel libre : Linus Torvalds. À cette époque, cet étudiant finlandais de l'université d'Helsinki cherche à écrire un noyau, selon la légende pour pouvoir utiliser sur son ordinateur personnel les programmes sur lesquels il travaille dans le cadre de ses études. II a alors l'idée brillante de rendre disponible sur Internet son travail inachevé, et d'inciter les informaticiens qui le souhaitent à le compléter. Grâce aux listes de diffusion et aux forums électroniques, des centaines puis des milliers de programmeurs en viennent à unir leurs efforts pour développer ce noyau, entre-temps nommé Linux. Bientôt, la combinaison des logiciels GNU et du noyau Linux donne naissance à un système d'exploitation complet : GNU/Linux, plus couramment appelé Linux.

Celui-ci a aujourd'hui acquis une solide réputation de fiabilité, et est devenu le concurrent principal de Windows. II est emblématique de la réussite du logiciel libre, dont témoignent également Firefox, Apache, Open Office, et bien d'autres. Au fil des ans, ces succès ont éveillé l'intérêt des géants du secteur informatique, et ont favorisé une pénétration croissante des logiques économiques dans le monde du libre. Un exemple significatif est celui d'IBM. Alors que l'entreprise est en difficulté, ses dirigeants décident en 1999 de rendre libres de grandes quantités de lignes de code propriétaires, et de mettre en place des équipes pour travailler sur les projets Apache et Linux. Ce soutien à des projets libres est une réussite, et il s'amplifie à partir de 2002. Les bénéfices qu'en retire IBM sont en effet multiples : économies substantielles ${ }^{4}$, réorientation de son activité vers de nouvelles offres de service $^{5}$, amélioration de son image...

\footnotetext{
${ }^{2}$ Le « logiciel libre » s'oppose ainsi au « logiciel propriétaire ». On insistera sur le fait que c'est bien cette opposition qui est pertinente, et non l'opposition entre logiciel libre et logiciel commercial. Comme le répète souvent Richard Stallman, "free software" is a matter of liberty, not price. To understand the concept, you should think of "free speech", not "free beer" " (http://www.gnu.org/philosophy/free-sw.html).

${ }^{3}$ Comme son nom l'indique, "le noyau d'un système d'exploitation est le cœur du système, qui s'occupe de fournir aux logiciels une interface pour utiliser le matériel ». Source : Wikipedia, http://fr.wikipedia.org/wiki/Noyau_Linux.

${ }^{4}$ IBM, qui investit 100 millions de dollars par an pour le développement de Linux, estime qu'il lui faudrait un investissement dix fois supérieur pour développer seul un système d'exploitation équivalent (Tapscott et Williams, 2007, pp. 93 et 97).

${ }^{5}$ Par exemple, la formation aux logiciels libres, ou l'installation et la personnalisation de systèmes libres.
} 


\section{Sébastien BROCA}

\subsection{L'idéologie du Libre}

L'influence croissante des grands groupes informatiques a suscité d'importantes réserves et révélé quelques fractures parmi les défenseurs du logiciel libre. En témoigne notamment la controverse ayant éclaté à la fin des années 1990, suite au lancement de l'Open Source Initiative. Cette organisation est créée en 1998 pour promouvoir un label dissident (OSI approved), censé être moins contraignant que la licence GPL, et donc plus attractif pour le monde des affaires. L'initiative est condamnée par Richard Stallman et la Free Software Foundation, bien que dans les faits, la différence entre les deux labels devienne assez rapidement de pure forme ${ }^{6}$. Le débat - parfois virulent - entre les deux parties met en lumière la coexistence au sein du monde du libre de deux "philosophies» assez nettement opposées. Pour les partisans de l'open source, les logiciels libres doivent êtres défendus pour l'unique raison qu'ils sont meilleurs que les logiciels propriétaires ! À l'inverse, pour les défenseurs du free software et son fondateur Richard Stallman, la performance technologique est une préoccupation secondaire par rapport au mouvement social que représente le logiciel libre, et aux principes qu'il défend.

Par-delà son contenu, la controverse entre open source et free software révèle l'importance des discours de positionnement idéologique dans le milieu du libre. Le mouvement du logiciel libre est ainsi indissociable des discours produits par ses acteurs pour légitimer et promouvoir leur pratique de la programmation informatique. Ce trait est particulièrement marquant, s'agissant de la question de l'organisation des communautés de développeurs. Celles-ci sont souvent opposées aux structures pyramidales dominantes dans les sphères économiques et politiques (grandes entreprises, partis politiques, etc.). Elles sont décrites comme mettant en œuvre une organisation horizontale, reposant sur le partage de l'information et la coopération directe entre participants. Seuls les individus affectueusement nommés « dictateurs bienveillants " sont censés y tenir un rôle hiérarchique. II s'agit d'une ou plusieurs personnes qui, en fonction du mérite qui leur est reconnu par la communauté, assurent pour chaque projet une fonction de direction, de coordination, et de sélection des contributions. Au sein du projet Linux, Linus Torvalds dirige ainsi la cellule chargée de choisir et d'assembler les modifications apportées au noyau du code source. À cette restriction près, l'idéal véhiculé par les partisans du logiciel libre est bien celui d'une

\footnotetext{
${ }^{6}$ Aujourd'hui, il est ainsi bien difficile de trouver un projet open source qui ne soit pas free software, et inversement. Les deux dénominations sont d'ailleurs le plus souvent employées indifféremment dans les médias. Dans le présent article, nous utilisons l'expression «logiciel libre » de façon générique, pour désigner à la fois les projets labellisés free software et les projets open source.
} 
Du logiciel libre aux théories de l'intelligence collective

communauté d'égaux, reposant sur le partage, la collaboration, et le jugement par les pairs.

Cette image est toutefois un peu trop parfaite pour ne pas fournir un reflet quelque peu déformé de la réalité. II faut ainsi prendre garde à ce que le discours des défenseurs du logiciel libre peut avoir de militant, voire d'idéologique. Certaines études de terrain semblent ainsi démontrer que les structures hiérarchiques sont souvent plus fortes que ce que les acteurs euxmêmes veulent bien admettre. Dans un travail réalisé en 2004, Thomas Basset montre ainsi, en étudiant le développement de la suite logiciels VideoLAN ${ }^{7}$, qu'il existe un décalage important entre le discours des participants au projet et la réalité des pratiques. Bien que les développeurs mettent en avant l'idéal d'un «libre échange du savoir entre personnes égales [...] hors de toute structure hiérarchique » (Basset, 2003, p. 28), l'observation du chercheur met en lumière l'existence d'une forte hiérarchie informelle. Au moment de l'étude, le projet VideoLAN repose ainsi sur une distribution du travail très inégalitaire, encore renforcée par des "manœuvres volontaires de rétention de l'information" (Basset, 2003, p. 52) en contradiction flagrante avec les discours tenus.

De façon générale, il faut avoir en tête que le mouvement du logiciel libre a non seulement produit des réalisations de tout premier ordre (Linux. Apache, etc.), mais aussi - et c'est là presque aussi important - un ensemble de discours mettant en avant certaines valeurs et certains modes de fonctionnement. Or, ces discours fonctionnent parfois comme une véritable idéologie, contribuant à voiler la réalité des pratiques. Par ailleurs, s'il est vrai que cette idéologie du libre n'est pas parfaitement homogène et cohérente (c'est ce qui apparaît notamment à travers la controverse entre free software et open source mentionnée plus haut), il s'en dégage néanmoins certaines constantes : méfiance envers la hiérarchie, valorisation du mérite individuel, promotion d'une éthique de la collaboration. On insistera ainsi sur le fait que la valorisation simultanée du mérite individuel et de la collaboration n'est contradictoire que superficiellement. Le mode d'organisation des communautés du libre est précisément censé permettre à chacun de concilier une grande autonomie dans son travail avec une inscription dans un projet collectif. En effet, une organisation horizontale apparaît par définition peu susceptible de soumettre les individus au groupe, en ce qu'elle refuse toute forme de hiérarchie et de contrôle centralisé. Dans le même temps, elle permet à un grand nombre de relations de

\footnotetext{
${ }^{7}$ Le développement de la suite logiciels VideoLAN, dont est issu le célèbre lecteur vidéo VLC, a été initié en 1995 par des élèves de l'École Centrale Paris (ECP). Depuis 2001, il s'agit d'un projet libre réalisé sous licence GPL, mené conjointement par des élèves de l'ECP et par des développeurs extérieurs.
} 
coopération de se nouer, dans la mesure où tout le monde peut potentiellement être en contact avec tout le monde. Si l'on suit l'idéologie du libre, il faut donc dire que la communauté, pour autant qu'en soient bannies les rigidités hiérarchiques et qu'y soit favorisée la collaboration, se présente comme le terrain le plus propice à révéler le mérite individuel. Que les choses soient plus compliquées en pratique est une évidence qu'il faut garder en tête. Néanmoins, il n'est pas exagéré d'affirmer que le mouvement du logiciel libre véhicule in fine une certaine idée des structures les plus aptes à assurer le plein épanouissement de l'individu et de la collectivité, dans le domaine de la programmation informatique voire au-delà.

\section{Le logiciel libre comme modèle social d'avant-garde}

Je voudrais désormais pousser le raisonnement plus loin, et examiner la manière dont certains intellectuels n'étant pas directement impliqués dans le milieu du logiciel libre, se sont emparés de cette thématique pour fonder leurs analyses d'un certain nombre de transformations sociales en cours. Autrement dit, je voudrais considérer des discours qui vont au-delà de ce que j'ai nommé l'idéologie du logiciel libre, dans la mesure où ils se présentent comme porteurs d'une analyse de portée générale sur l'évolution de nos sociétés. Ces discours tendent ainsi à reprendre l'idéalisation du logiciel libre véhiculée par ses acteurs, tout en étendant la portée du modèle à d'autres activités sociales. Le logiciel libre devient de la sorte un socle sur lequel se trouve bâtie toute une construction intellectuelle. Je développerai trois exemples pour appuyer ce propos.

\subsection{Pekka Himanen : la propagation de "l'éthique hacker 》}

Le philosophe finlandais Pekka Himanen a consacré à " l'éthique hacker » un ouvrage au retentissement important, paru en France en 2001: L'Éthique hacker et l'esprit de l'ère de l'information. ${ }^{8}$. II y soutient que les pratiques et les valeurs du monde du logiciel libre ont donné naissance à « une nouvelle éthique du travail qui s'oppose à l'éthique protestante du travail telle que l'a définie Max Weber » (Himanen, 2001, p. 10). Cette nouvelle éthique se caractériserait par une relation au travail fondée sur la passion et l'intérêt personnel, et non sur le devoir moral et l'intérêt financier. Pour les hackers, le travail ne serait ainsi ni

\footnotetext{
8 Il convient de préciser que le terme «hacker» n'est pas employé par l'auteur en son sens médiatique de « pirate informatique », mais bien en son sens originel, c'est-à-dire comme désignant tous les véritables passionnés d'informatique, et en premier lieu les partisans du logiciel libre.
} 
Du logiciel libre aux théories de l'intelligence collective

posé comme fin en soi indépendamment de son contenu, ni considéré comme simple moyen d'assurer sa subsistance ou sa richesse. L'important serait au contraire la satisfaction personnelle éprouvée dans la réalisation d'une tâche, devant être vécue comme intrinsèquement intéressante et gratifiante : «Les hackers font de la programmation parce que les défis qu'elle génère ont un intérêt intrinsèque pour eux " écrit ainsi P. Himanen (Ibid., p. 23). II cite également Linus Torvalds, qui affirme que pour lui « Linux a largement été un hobby (mais un sérieux, le meilleur de tous) » (Ibid., p. 34).

Ce nouveau rapport au travail, qui repose sur une logique de développement de soi (Selbstentfaltung ${ }^{9}$ ), irait de pair avec un nouveau rapport au temps. Ainsi, pour les hackers, la distinction entre temps de travail et temps de loisir se trouverait brouillée, au profit d'un temps flexible où travail, hobbies, familles, collègues et amis se trouveraient sans cesse mélangés.

À la lumière de ces éléments, on peut mettre en doute la radicale nouveauté de cette «éthique hacker ». $P$. Himanen reconnaît du reste lui-même que les universitaires ou - d'une manière différente - les artistes, entretiennent depuis longtemps ce même rapport passionné à leur travail, et valorisent eux aussi une organisation relativement «libre » de leur temps. La véritable nouveauté résiderait en fait dans la manière dont ce rapport au travail serait en train de se répandre dans la société, à partir des milieux hacker.

[...] l'éthique hacker du travail se propage doucement vers d'autres secteurs, à l'image de l'éthique protestante qui, selon Weber, a fait son chemin en partant des entreprises créées par des Protestants pour finir par dominer l'esprit du capitalisme (Ibid., pp. 66-67).

Autrement dit, à la faveur du passage de la société industrielle à la " société en réseaux » (Castells, 1998) ${ }^{10}$, le rapport au travail des hackers deviendrait viable pour de larges pans de la main d'œuvre. Leur éthique se substituerait ainsi progressivement à l'éthique protestante traditionnelle (ou à sa déclinaison contemporaine, érigeant l'argent en valeur suprême). Les hackers seraient finalement une sorte de groupe social d'avant-garde, et le mouvement du

\footnotetext{
${ }^{9}$ Ce terme, parfois proposé pour rendre compte du rapport des développeurs à leur travail, semble assez pertinent, dans la mesure où il rend compte du fait que les motivations des hackers sont essentiellement personnelles, mais néanmoins nullement réductibles à un simple calcul économique (Merten, 2002).

${ }^{10}$ Pekka Himanen s'inspire largement des analyses de Manuel Castells, qui a développé dès 1996 l'idée selon laquelle un nouveau modèle social serait en train d'émerger à partir d'Internet et de la figure du réseau. M. Castells est par ailleurs l'auteur de l'« épilogue » de l'ouvrage de P. Himanen.
} 


\section{Sébastien BROCA}

logiciel libre la matrice dont émergerait une nouvelle société. "Le modèle hacker ouvert pourrait se transformer en un modèle social " écrit P. Himanen (2001, p. 86).

Cette thèse n'est pas sans poser certaines difficultés. En effet, la possibilité de généraliser la relation au travail des hackers à d'autres groupes sociaux, et à d'autres secteurs économiques, est loin d'être évidente. Pekka Himanen s'expose ainsi au reproche d'ethnocentrisme, dans la mesure où tous les types d'emplois ne semblent pas susceptibles d'engendrer un rapport passionné au travail. Ne se rend-il pas coupable de généraliser une attitude, qui est en fait un privilège réservé à une partie infime de la population ? N'oublie-t-il pas un peu vite la très grande spécificité des hackers en tant que catégorie socioprofessionnelle, lorsqu'il affirme que leur éthique a une valeur quasi « universelle » (Ibid., p. 26) ?

La thèse d'Himanen d'une propagation de l'éthique hacker dans l'ensemble du corps social apparaît relativement difficile à maintenir telle quelle. On peut alors choisir de la tirer du côté de l'utopie, c'est-à-dire d'un état idéal du social dans lequel le travail ne serait plus aliénation mais autodéploiement. Mais tel n'est certainement pas le propos de l'auteur, qui adopte dans son ouvrage une posture plutôt « sociologique ". Peut-être faut-il alors se résoudre à tirer de son ouvrage - et à l'encontre de ce qu'il écrit lui-même - une thèse plus " faible ». La mise en pratique de l'éthique hacker ne serait finalement possible que dans des secteurs très spécifiques, pour les professionnels de l'information, les artistes, ou les chercheurs. La généralisation à l'ensemble de la société de l'attitude au travail propre au milieu du logiciel libre apparaît en effet assez peu vraisemblable.

\subsection{Yann Moulier Boutang : le logiciel libre comme modèle productif d'un nouveau capitalisme}

L'exemple du logiciel libre est également tout à fait central dans la démarche de Yann Moulier Boutang, économiste et directeur de la revue Multitudes. Celuici a entrepris depuis quelques années, en collaboration étroite avec d'autres chercheurs (Antonella Corsani, Carlo Vercellone, Bernard Paulré...), de renouveler les cadres d'analyse de la science économique pour saisir les mutations contemporaines du capitalisme. Ce projet a donné naissance à l'hypothèse du "capitalisme cognitif ». Selon Yann Moulier Boutang, nous serions ainsi en train de sortir du capitalisme industriel pour entrer dans un nouveau type d'économie, "fondé sur l'accumulation du capital immatériel, la diffusion du savoir et le rôle moteur de l'économie de la connaissance " (Moulier Boutang, 2007, p. 85). Le capitalisme ne se nourrirait plus « du muscle 
Du logiciel libre aux théories de l'intelligence collective

consommé dans les machines marchant à la dissipation de l'énergie "carbofossile" » (Ibid., p. 65), mais de la « force cognitive collective » (Ibid., p. 65), ou encore de «l'intelligence collective » (Ibid., pp. 61-67). La source de la richesse résiderait donc aujourd'hui dans le travail social de communication et d'invention, ou encore dans la manipulation et la création de connaissances.

À mesure que les formes du travail et les sources de la richesse se modifieraient, le mode de production propre au capitalisme industriel entrerait en crise. Certes bien loin de disparaître complètement, il serait néanmoins délaissé dans les secteurs « avancés » de l'économie. En effet, l'adaptabilité, la flexibilité et la créativité permises par l'organisation en réseau, se révèleraient plus propres à révéler l'intelligence collective, que la rigidité de la one best way propre au taylorisme.

La thèse de Yann Moulier Boutang est ainsi qu'un nouveau mode de production émerge, qu'il définit comme "le travail de coopération des cerveaux réunis en réseau au moyen d'ordinateurs » (Ibid., p. 95). Point n'est besoin de trop de perspicacité pour s'apercevoir que cette description correspond parfaitement au modèle de fonctionnement des communautés du logiciel libre. Et pour cause ! Selon Yann Moulier Boutang, ces communautés sont l'exemple paradigmatique de ce nouveau mode de production propre au capitalisme cognitif. Ainsi, « le phénomène social et économique du libre » fournirait

un véritable modèle productif. Et ceci, tant sur le plan des forces sociales nouvelles que l'on peut repérer, sur celui de la division sociale du travail, que sur celui de la rationalité des agents économiques qui se trouve ainsi inventée, promue, des formes d'identité non pas au travail mais à un travail qui a changé fortement de contenu (Ibid., p. 125).

Yann Moulier Boutang accorde ainsi au logiciel libre une valeur d'exemplarité : ce qu'ont réussi à mettre en place quelques milliers de passionnés à travers le monde dans le domaine de la production informatique, serait un avant-goût d'un bouleversement global de l'organisation du travail. Suivant la voie tracée par les hackers, les forces productives deviendraient progressivement comparables à "l'activité collective cérébrale mobilisée en réseaux numériques interconnectés » (Ibid., p. 93).

Dans le même temps, le capitalisme cognitif deviendrait de plus en plus assimilable à une économie open source. En effet, dès lors que la richesse 


\section{Sébastien BROCA}

résiderait désormais dans l'ensemble du travail social de communication et d'invention, l'entreprise n'en serait plus l'unique productrice. Elle se devrait au contraire de capter une richesse antérieure, c'est-à-dire de valoriser ce qui émerge spontanément de l'ensemble des échanges sociaux.

$$
\begin{aligned}
& \text { L'intelligence entrepreneuriale consiste désormais à } \\
& \text { convertir la richesse déjà là dans l'espace virtuel du } \\
& \text { numérique en valeur économique (lbid., p. 167). }
\end{aligned}
$$

Les entreprises auraient ainsi un intérêt profond à laisser se développer sans entraves la coopération en réseau, car celle-ci leur offrirait in fine les meilleures occasions de profit, en leur permettant de tirer profit d'une grande quantité de travail gratuit.

L'activité humaine innovante de la coopération des cerveaux à l'ère numérique produit dans la science, dans l'art, dans les formes collectives du lien social des gisements nouveaux et impressionnants d'externalités positives pour les entreprises, c'est-àdire de travail gratuit incorporable dans de nouveaux dispositifs de captation et de mise en forme (lbid., p. 122).

Comme l'aura déjà remarqué le lecteur attentif, ce nouveau business model correspond en tout point aux stratégies mises en œuvre par certaines grandes multinationales du secteur informatique pour tirer parti du logiciel libre. Ainsi, quand IBM profite du travail gratuit des communautés Linux ou Apache, et réoriente ainsi son activité vers une nouvelle offre de services (cf. supra), l'entreprise opère précisément ce que Yann Moulier Boutang décrit comme une captation de l'intelligence collective.

Le logiciel libre constitue donc pour Yann Moulier Boutang le phénomène économique et social, permettant d'analyser le capitalisme cognitif dans ses multiples facettes. Les communautés de développeurs du libre auraient ainsi «inventé » le mode de production caractéristique de ce nouveau régime économique, tandis que les entreprises ayant investi dans l'open source auraient développé son business model. La démarche théorique de Yann Moulier Boutang apparaît donc finalement très proche de celle de Pekka Himanen, dans la mesure où elle procède par généralisation à partir de l'exemple des communautés du logiciel libre, en tendant à faire de celles-ci des sortes d'avant-gardes. Elle s'expose de ce fait au même type de critique. Emporté par son admiration pour « la révolution californienne du capitalisme » 
Du logiciel libre aux théories de l'intelligence collective

(Ibid., p. 25), Moulier Boutang semble sous-estimer la spécificité du logiciel libre, et la difficulté à exporter ce modèle vers d'autres secteurs économiques. Fabien Granjon a ainsi noté que "les nouveaux aspects de production "sur lesquels les tenants de la thèse du " capitalisme cognitif » fondent leur théorie, "ne constituent que des sphères relativement restreintes de l'activité économique : ainsi en est-il du domaine du logiciel libre » (Granjon, 2008, p. 63). De manière analogue, Michel Husson a pointé la tendance de ceux qu'ils nomment les « cognitivistes" à " extrapoler des tendances partielles sans comprendre qu'elles ne peuvent se généraliser » (Husson, 2007, p. 139).

Il est intéressant de noter que Yann Moulier Boutang est conscient de s'exposer à de telles objections. II tente d'y répondre, en proposant une analogie entre sa démarche et celle de Marx à l'aube de l'ère industrielle :

On s'intéresse en général à des observations empiriques sélectionnées dans un fatras rhapsodique d'informations multiples parce qu'on cherche les variables pertinentes qui commandent la tonalité d'ensemble ou permettent de prévoir des trajectoires d'évolution. Le grand trait de génie de Marx et Engels n'est pas d'avoir étudié la population laborieuse la plus nombreuse en Angleterre, (c'étaient les domestiques qui se comptaient par millions) mais les quelques 250 000 ouvriers des usines de Manchester (Moulier Boutang, 2007, p. 99).

L'argument est malicieux, et non dénué de pertinence. Toutefois, il ne suffit pas à effacer l'impression que la généralisation opérée par Moulier Boutang à partir du logiciel libre repose sur un pari. Celui d'affirmer que nos économies post-industrielles vont effectivement suivre la voie tracée par les expériences pionnières du logiciel libre. Or, s'il y a là un avenir possible, ce n'est certainement pas le seul ! La période actuelle paraît en effet trop incertaine, pour qu'on puisse écarter, par exemple, l'hypothèse du développement d'un "capitalisme gestionnaire d'effets de réseaux et de verrouillage " (Aigrain, 2007 , p. 252), capitalisme qui serait bien entendu totalement contraire au modèle promu par les tenants du libre. Yann Moulier Boutang ne semble certes pas considérer ce scénario comme crédible, mais c'est là une position qui relève davantage de l'acte de foi que de la démonstration irréfutable. Ou, pour le dire de façon plus clémente : Yann Moulier Boutang assume les risques et les faiblesses de tout discours sur le social ne se contentant pas d'être descriptif, mais ayant une double visée de synthèse et de prospective. Sa démarche visant 
Sébastien BROCA

à faire du logiciel libre le modèle d'un nouveau capitalisme en gestation est donc nécessairement très perméable à la critique.

\subsection{Michael Hardt et Antonio Negri : la démocratie comme "société open source ".}

Ayant bénéficié d'un important succès commercial, les ouvrages Empire et Multitudes de Michael Hardt et Antonio Negri se présentent comme une tentative de rénover la philosophie politique critique, à l'heure de la mondialisation et des nouvelles technologies de l'information et de la communication. Ils sont notamment consacrés à la construction d'une théorie originale de la démocratie, inspirée en partie par le mouvement du logiciel libre. Avant d'aborder celle-ci en tant que telle, il faut préciser que les auteurs ancrent leur réflexion dans une analyse des transformations économiques contemporaines ; analyse tout à fait semblable à celle de Yann Moulier Boutang. Ils insistent ainsi sur la progression de la part du travail immatériel, et sur l'importance nouvelle de la coopération en réseau sur le modèle du logiciel libre.

L'originalité de leur propos se situe dans la thèse selon laquelle ces transformations économiques rejailliraient sur toutes les dimensions de la vie en société. C'est ce qu'ils appellent, en reprenant un terme forgé par Michel Foucault, la dimension biopolitique de la production.

Dans le cadre de ce travail immatériel, la production déborde hors des frontières traditionnelles de l'économie et se déverse directement dans le domaine culturel, social et politique. Elle crée non seulement des biens matériels, mais des relations sociales concrètes et des formes de vie. Nous appelons "biopolitique " cette forme de production, afin de souligner le caractère générique de ses produits et le fait qu'elle a directement prise sur l'ensemble de la vie sociale (Hardt et Negri, 2004, p. 121).

Ce caractère biopolitique de la production est, selon les auteurs, à l'origine d'une isomorphie croissante entre les structures économiques et les autres structures sociales, y compris politiques. Autrement dit, le développement de la forme réseau dans le cadre de la production immatérielle aurait pour corrélat la progression de cette même forme dans toutes les dimensions de la vie sociale. Ainsi Michael Hardt et Antonio Negri écrivent-ils notamment que 


\begin{abstract}
les institutions de la démocratie doivent aujourd'hui coïncider avec les réseaux communicatifs et collaboratifs qui ne cessent de produire et reproduire la vie sociale (lbid., p. 400).
\end{abstract}

C'est dans ce cadre conceptuel qu'il faut comprendre le lien établi par les auteurs entre le mouvement du logiciel libre et la redéfinition de la démocratie qu'ils appellent de leurs voeux. On peut ainsi - moyennant une simplification acceptable - donner à leur raisonnement la forme d'un syllogisme : Structures économiques et structures politiques tendent à devenir semblables. Or, le logiciel libre est emblématique des nouvelles structures économiques. Donc, le logiciel libre devient emblématique des nouvelles structures politiques ! Michael Hardt et Antonio Negri écrivent ainsi :

On peut donc voir la démocratie de la multitude comme une société open source, c'est-à-dire une société dont le code source est révélé, permettant à tous de collaborer à la résolution de ses problèmes et de créer des programmes sociaux plus performants (Ibid., p. 385).

À travers l'expression de "société open source ", Michael Hardt et Antonio Negri poussent la généralisation à partir du mouvement du logiciel libre à l'extrême. Ils accordent à celui-ci une place centrale dans la construction d'un nouveau modèle de société, susceptible de fournir une alternative crédible au libéralisme débridé et aux "vieilles " démocraties parlementaires. Hardt et Negri font ainsi du projet du logiciel libre l'horizon d'une certaine gauche radicale : la "société open source » et la "démocratie de la multitude " sont fondues en un seul et même objectif politique.

Évidemment, c'est là prêter énormément au mouvement du logiciel libre (ici confondu avec l'open source), et faire peu de cas des ambiguïtés politiques qu'il recèle. On remarquera ainsi que les grands principes du libre (libre partage de l'information, organisation horizontale, autonomie laissée à chacun) dessinent en fait un projet politique relativement modeste. Comme le remarque Patrice Riemens, nous sommes "très en deçà des exigences de justice, d'équité, d'égalité, d'émancipation » (Riemens, 2002, p. 185), qui animent la plupart des grands projets politiques de gauche. De surcroît, il paraît délicat - plus en tous les cas que Negri et Hardt ne le laissent supposer - de faire du libre un projet politique global. En effet, l'engagement social d'une majorité de hackers semble se limiter à un attachement à la disponibilité du code au sein du monde informatique. II a d'ailleurs été remarqué que 


\section{Sébastien BROCA}

l'étendue du spectre des opinions politiques entretenues par les hackers individuels [...] est surprenante, et totalement inimaginable dans quelque autre « mouvement social » (lbid., p. 185).

II semble donc que Michael Hardt et Antonio Negri accordent au logiciel libre une portée sociale et politique légèrement excessive. Ils participent en cela d'un mouvement plus général, qui a vu plusieurs intellectuels de gauche s'enthousiasmer pour le libre au cours des années $2000^{11}$. II est toutefois possible de faire une lecture légèrement différente de la référence à l'open source chez Hardt et Negri. On émettra ainsi l'hypothèse que leur intérêt se situe à un niveau plus abstrait. Autrement dit, la portée politique effective du mouvement du logiciel libre les intéresse peut-être moins, que le fait qu'il fournisse un modèle inédit pour penser une nouvelle forme et un nouveau contenu de la démocratie ${ }^{12}$. C'est donc à ce niveau, disons plus « théorique », que je voudrais situer ma dernière critique.

Pour l'énoncer succinctement, celle-ci consiste à dire que la mise en avant du modèle du libre pour (re)penser la question démocratique est porteuse d'une négation de la spécificité de la politique. Ainsi, si l'on prend au sérieux la référence à l'open source, la « démocratie de la multitude » aurait pour objet la production collaborative d'une forme d'expertise collective. Elle aurait à mettre en œuvre des processus d'agrégation de savoirs locaux. Elle se présenterait comme une sorte de gigantesque entreprise de débogage, ou comme un problem solving à grande échelle.

De telles idées ont certes des aspects séduisants, d'une part parce qu'elles semblent promettre une gestion politique plus efficace ${ }^{13}$, d'autre part parce qu'elles renouent avec l'idéal d'une démocratie qui reposerait véritablement sur la participation effective de tous et bannirait le secret. Toutefois, l'analogie entre l'open source et la démocratie pose des difficultés majeures. Ainsi, dans le cas du logiciel libre, la finalité de la collaboration ne prête pas à discussion, puisqu'il s'agit tout simplement de produire le meilleur logiciel possible. Corrélativement, la réussite (ou l'échec) des diverses modifications souffre assez peu de

\footnotetext{
${ }^{11}$ Citons par exemple André Gorz, pour qui « la communauté virtuelle, virtuellement universelle des usagers-producteurs de logiciels et de réseaux libres, instaure des rapports sociaux qui ébauchent une négation pratique des rapports sociaux capitalistes » (Gorz, 2003, p. 93) .

${ }^{12} \mathrm{Ce}$ choix de lecture se trouve corroboré par le faible nombre d'éléments factuels sur le logiciel libre présents dans l'ouvrage de Michael Hardt et Antonio Negri.

${ }_{13} \mathrm{Si}$ l'on accepte l'analogie entre société et logiciel, on peut effectivement penser qu'une « démocratie open source » résoudrait nos problèmes sociaux plus efficacement que nos démocraties parlementaires, de la même façon que les logiciels libres s'avèrent souvent plus performants que les logiciels propriétaires...
} 
Du logiciel libre aux théories de l'intelligence collective

contestation. Elle s'évalue aisément, puisqu'il suffit d'exécuter le code pour observer si la nouvelle mouture est plus rapide et plus complète que l'ancienne, si elle contient des bugs, etc. En matière de démocratie, les choses sont nettement plus compliquées. Ainsi, il y a rarement unanimité sur l'identification et la hiérarchisation des "problèmes" auxquels la collectivité se trouve confrontée. On peut même dire que la discussion sur les fins de l'action politique est inéliminable : celles-ci sont toujours objets de litige. Le fonctionnement démocratique ne peut par conséquent être réduit à la recherche collective des moyens les plus efficaces de résoudre des problèmes, qui seraient eux soustraits à toute forme de mise en question.

Tout régime politique ouvert ne cesse ainsi d'être confronté à des questions, que l'acquisition d'un savoir, quel qu'il soit, ne peut suffire à trancher. La démocratie ne saurait la plupart du temps se contenter d'une somme de connaissances objectives pour déterminer sa pratique. Elle vit au contraire de la confrontation d'une diversité d'engagements argumentés, mettant en jeu une pluralité de valeurs, d'intérêts ou de " choix de société ". Elle est indissociable d'un régime de rationalité particulier : l'échange d'opinions conclu par le vote à la majorité, et non pas la production collaborative de connaissances dont dériveraient mécaniquement les décisions.

La référence à une hypothétique "démocratie open source " importe donc indûment dans le champ politique la rationalité technique et scientifique, au détriment de la rationalité proprement politique. Autrement dit, elle néglige le rapport de la politique à l'opinion, considérée en tant que catégorie épistémologique. Ainsi, dans le recouvrement de la doxa par l'épistèmê, et dans le remplacement du débat contradictoire par la collaboration en réseau, ce serait bien l'essence des enjeux et des procédures démocratiques qui serait perdue.

\section{Conclusion}

Au terme de ce parcours, il apparaît que les pratiques mises en place par les communautés du logiciel libre sont sujettes à une double distorsion. Elles se trouvent tout d'abord fréquemment idéalisées par les acteurs du libre euxmêmes, dont le discours militant a souvent pour effet de recouvrir les difficultés posées par la mise en application du modèle qu'ils défendent. Une deuxième distorsion est imputable à certains intellectuels qui, en conférant aux expériences du logiciel libre une portée social générale, minimisent la spécificité 


\section{Sébastien BROCA}

d'un mouvement dont les enjeux sont - avant tout, bien que non exclusivement - internes au secteur informatique ${ }^{14}$.

Ces théories «inspirées" du logiciel libre présentent des similitudes frappantes. Par-delà leurs champs d'application privilégiés, elles s'accordent pour voir la spécificité de notre période dans le développement de nouvelles formes de collaboration « intelligentes » médiatisées par Internet. À les suivre, les changements que connaît notre époque - qu'il s'agisse des mutations des formes de socialité, des bouleversements économiques, ou encore des nouvelles formes de l'engagement politique - seraient directement imputables à la nouvelle configuration sociotechnique, permettant de « mettre en réseau les cerveaux ». Ces changements seraient révélateurs d'une période de transition, entre une société industrielle mourante, et une société de "l'intelligence collective » propulsée par les nouvelles formes d'échange et de production de connaissances.

Si ces théories saisissent indéniablement un trait marquant de notre époque, elles semblent néanmoins pêcher par un certain réductionnisme. Elles présentent en effet une tendance à ramener des phénomènes hétérogènes à une grille d'explication unique, faisant fi des spécificités des différents domaines de la vie sociale. Elles tendent parfois aussi à occulter des traits particulièrement frappants de notre époque: montée inouïe des inégalités, déséquilibres écologiques, etc. On pourra ainsi regretter que le discours sur le partage de l'information ne s'accompagne que trop rarement d'un discours sur le partage des richesses, ou des ressources naturelles.

\footnotetext{
${ }^{14}$ Précisons que si nous avons - pour la clarté de l'exposé - distingué ces deux types de discours, cette séparation n'a bien entendu rien d'étanche. Ainsi, les intellectuels fondant leur démarche théorique sur l'exemple du logiciel libre sont imprégnés d'un certain discours militant. On peut aussi remarquer qu'il y a déjà dans l'idéologie du Libre une tendance à vouloir accentuer la portée sociale du « modèle ouvert ", tendance que certains intellectuels s'empressent ensuite de reprendre et de développer.
} 
Du logiciel libre aux théories de l'intelligence collective

\section{Références bibliographiques}

AIGRAIN P., 2007, «Capitalisme cognitif et politiques qualitatives après la révolution informationnelle » in MOULIER BOUTANG Y., 2007, Le capitalisme cognitif, Paris, Éditions Amsterdam.

BASSET T., 2003, Monographie d'un logiciel libre : VideoLAN, Mémoire de DEA, Institut d'Études Politiques de Paris, en ligne : http://www.framasoft.net/IMG/videolan.pdf (consulté le 19 novembre 2008).

CASTELLS M., 1998, L'ère de l'information, vol. 1 : La société en réseaux, Paris, Fayard.

GORZ A., 2003, L'immatériel, Paris, Galilée.

GRANJON F., 2008, « Les nouveaux résistants à l'ère du numérique. Entre utopie sociale et déterminisme technique », in PROULX S. et alii, 2008, L'action communautaire québecoise à l'ère du numérique, Montréal, Presses de l'Université du Québec, pp. 59-76.

HARDT M. et NEGRI A., 2004, Multitudes, Paris, La Découverte.

HIMANEN P., 2001, L'éthique hacker et l'esprit de l'ère de l'information, Paris, Exils.

HUSSON M., 2007, « Notes critiques sur le capitalisme cognitif », ContreTemps, n918, pp. 138-141, en ligne : http://hussonet.free. fr/cognict.pdf (consulté le 10 novembre 2008).

MERTEN S., 2002, « Logiciel libre et éthique du développement de soi : entretien avec Joanne Richardson », Multitudes, n, pp. 188-199, en ligne : http://multitudes.samizdat.net/Logiciel-libre-et-ethique-du (consulté le 19 novembre 2008).

MOGLEN E., 2001, «L'anarchisme triomphant : le logiciel libre et la mort du copyright », Multitudes, $n^{\circ} 5$, pp. 146-183, en ligne: http://multitudes.samizdat.net/L-anarchisme-triomphant (consulté le 19 novembre 2008).

MOULIER BOUTANG Y., 2007, Le capitalisme cognitif, Paris, Éditions Amsterdam.

RIEMENS P., 2002, “Quelques réflexions sur le concept de "culture hacker” », Multitudes, $n^{\circ} 8, \mathrm{pp} .181-187$, en ligne : 


\section{Sébastien BROCA}

http://multitudes.samizdat.net/Quelques-reflexions-sur-le-concept (consulté le 19 novembre 2008).

TAPSCOTT D. et WILLIAMS A. D., 2007, Wikinomics, Paris, Pearson Education France. 